SPECIAL REPORT

\title{
EFFICACY OF LOW ENERGY NEUROFEEDBACK SYSTEM IN REHABILITATION OF MENTAL HEALTH DISORDERS
}

\begin{abstract}
The Low Energy Neuro feedback System (LENS) is one of the growing approaches used to bring Quality of life (QOL) to the patients suffering from mental health issues and Nervous system disorders by elevating the symptoms. It is a computer-based software program that is connected to EEG box which measure brain waves. This approach is different from Neuro feedback technique as it uses very low electromagnetic strength. It brings in noticeable improvement after few second of treatment session which does not require conscious awareness or voluntary participation from the client. It was first developed in the early 1900s by $\mathrm{Dr}$ Len Ochs to treat uncontrolled epilepsy, followed by many researches that signify its beneficence for treating many neurological and mental health disorders. This technique does not cure the disorder though it does elevate symptoms that improve the QOL. Although LENS is being exercise around the world, it is still unidentified by many professionals in Pakistan. Use of LENS adjacent to other therapies will bring revolution in Pakistan mental health rehabilitation. More research on LENS is suggested.
\end{abstract}

\section{KEYWORDS}

Brain waves, Low Energy Neuro feedback, Mental Health Disorders, EEG, Brain stimulation, Anosmia, Developmental Trauma, Quality of life.

\section{Saad Ali Mir}

Biomedical Engineering

Service Engineer

Biomadics

saadalimir.sk@gmail.com

\section{Fatima Khan}

Occupational Therapist Neelum Care Association Fatma_khan94@hotmail.com

[Mir SA, Khan F. Efficacy of Low Energy Neuro feedback System in Rehabilitation of Mental Health Disorders. Pak. j. rehabil. 2019;8(1):49-52] 


\section{INTRODUCTION}

The Low Energy Neurofeedback System (LENS) is one of the Electroencephalogram (EEG) biofeedback systems that have shown advantageous effects in the rehabilitation of many disorders such as central nervous system dysfunctions and mental health disorders. LENS was developed by a Californian psychologist; Dr. Len Ochs in the late 1960s after focusing first on animals followed by human research for treating uncontrolled epilepsy. It is an EEG biofeedback technique to retrain and recondition brain wave patterns (Kamlya, 201 1; Sterman, LoPresti, \& Fairchild, 2010) by placing one or more electrodes on the scalp and earlobes. The electronic equipment provides auditory and visual feedback about brain wave activity without inducing electric current to the brain'. It provides visual representation of brain waves activity to patient by detecting and amplifying the delicate electrical information from the patient's brain, which can then modify it.

\section{Brain Waves and NeuroFeedback Approaches}

Brainwaves are rhythmic or repetitive neural activity in the brain which is produced by synchronized electrical impulses generated by mass of neurons that communicate with each other ${ }^{2}$. It can also be observed by individual neuron either as oscillation in membrane potential or as rhythmic patterns of action potential that generates rhythmic activity at post synaptic neuron. Macroscopic oscillations arises from interaction between mass of neurons which can be observed by EEG which has different frequency from electrical impulses generated by the firing of individual neuron. These oscillations can be characterized by their frequency, amplitude and phase and crucial to the brain functioning such as thoughts and emotions ${ }^{2}$. The activity of these brain waves can be altered through Neurofeedback to bring on the desirable effects during rehabilitation of people suffering from mental health disorders ${ }^{3}$. The major difference between the brain waves is the magnitude of frequency. Discrepancy in frequency of brain waves is found in literature, the quoted magnitude shared is extracted from a book titled 'Quantitative EEG, Event-Related Potentials and Neurotherapy"4.

\section{Delta Waves (1 To $4 \mathrm{~Hz}$ )}

These waves are slow, have lowest frequency among others and penetrate deeply. It is generated in deepest meditation and disbands the external awareness. It stimulates healing and regeneration ${ }^{5}$.

\section{Theta Waves (4 To $8 \mathrm{~Hz}$ )}

These waves are generated in frontal lobe during sleep and deep meditation or relaxation. It controls subconscious part of the brain that retains memory, fears, nightmares and feelings6thus used in hypnosis and during REM sleep.

\section{Alpha Waves (8 To $12 \mathrm{~Hz}$ )}

Alpha brainwaves are dominant during quietly flowing thoughts, and in some meditative states. It is 'the power of now', being here, in the present. Alpha is the resting state for the brain that aid overall mental coordination, calmness, alertness, mind/body integration and learning ${ }^{7}$.

\section{Beta Waves (13 To $30 \mathrm{~Hz}$ )}

Beta brain waves are generated during alert state and high cognitive activities such as problem solving, judgment and decision making. It is produced more when the person is involved in doing task with more concentration and focus. High levels of Beta waves are associated with anxiety, disease, and feeling of separation, fight or flight ${ }^{8}$.

\section{Gamma Waves (38 To $80 \mathrm{~Hz}$ )}

It is fastest and high frequency brain wave which is involved in processing information from different parts of the brain. It modulates perception and consciousness, higher the magnitude of gamma waves will advance awareness and spiritual emergence?.

Growing literature suggest the significant effects of using EEG techniques for clinical intervention adjacent to medication as it is cost effective and non-invasive. Low Energy Neurofeedback is unique in itself as it does not only assist patients to condition healthier brainwave pattern but also helps in reorganizing brain physiology by providing very weak electromagnetic signals of strength 10-18 Watts/cm2, which is $1 / 400$ th the strength of input from the cellular phone10. LENS is different from conventional EEG biofeedback as it does not require patient's attention and awareness. It demands less time and learning of the client. It is unique and passive form of Neurofeedback.

\section{Working Principle of Lens Method}

The LENS uses very low power radio frequency signal to provide information back to the brain with the help of patient's EEG. The power is lesser than a digital watch, regardless of such a low power and weak signal method it produces quantifiable or measurable changes without patient's effort. The EEG sensors are placed on various points on the head and act as bi-directional channels or conduits for recording and measuring brain waves and then deliver a biofeedback signal through a computer software that slightly alter the signal called as "offset". The LENS method demands a patient to sit on a chair comfortably while a Neuro feedback practitioner applies a small EEG sensor with conductive paste to the scalp. By each sessions depending on the patient's treatment recovery, the process of measuring brain waves and creating a brain map occurs which will set out the purpose of creating a treatment plan, diagnosis and to compare the progress with the actual experience. The quantitative EEG recording is a brain wave and it is done from 21 points of the head. The course of the treatment results the formation of brain map and the doctor or practitioner will be looking for the areas under activity or suppressed in the brain wave patterns. On the 
basis of the brain map recordings and the signs and symptoms that patient report, a course of LENS therapy is chosen. The LENS treatment is consists of very weak radio frequency that is almost 3,000 times weaker that what brain is exposed to every time to cell phone. The feedback signal is extremely weak and the length of exposure is very short. The duration of feedback during a LENS session is from few seconds to 1 minute per side on the head.

\section{Benefits of Low Energy Neurofeedback}

The evidence from literature and researches supports the promising effects of LENS in elevating symptoms of mental health disorders, improving quality of living. An explorative study was conducted by Gillham.s and fellows to determine the efficacy of LENS for stress, anxiety and cognitive function of twenty medical students. The change of scores in Perceived Stress Scale (PSS) and Weschsler Abbreviated Scale of Intelligence (WASI) was recorded ${ }^{1 !}$.

\section{Developmental Trauma}

Any child who has experience chronic abuse, neglect or any other type of adversity while growing up are said to be victims of Developmental Trauma. Such children are at high risk of developing variety of mental health disorders such as post-traumatic stress disorder, anxiety, depression, increased aggression than their counterparts. Alteration in brainwaves is evident in such children. Application of LENS to such children showed improved psychotherapeutic response by modulating frequency of delta brain wave in frontal lobe ${ }^{12}$.

\begin{abstract}
Anger Control
Anger is a natural phenomenon characterized by resentment towards someone or something that one belief has done something wrong. Aggression has both positive and negative outcome as it motivates to find the solution of problem and take initiate against something wrong, but excessive anger can cause both physical and emotional problems.

Hammond (2010) and his colleagues studied the effects of LENS on elevating aggression by administering it four patients. Two of which showed significant improvement in controlling aggression as recorded on Sate-Trait Anger Expression Inventory-2. EEG finding of one of the patient showed significant modulation of Beta waves in frontal lobe and Theta waves in posterior and frontotemporal area of brain.
\end{abstract}

\section{Schizophrenia}

Schizophrenia is one of the most common mental illnesses. People suffering from this disorders experience illusions, restlessness, confusion, nonflexible muscles and delirium. Several researches signify the positive impact of Neurofeedback application ${ }^{13}$.

\section{Autism}

Autism spectrum Disorder is a developmental disorder that affects communication and behavior. The people with ASD have difficulty in communication and interaction with people, and have restricted interest and repetitive behavior. The literature shows that autistic children have abnormalities of alpha waves at early age and decrease in beta to alpha power ratio at the age of 22 to 25 years which can be associated with their irritability and restlessness ${ }^{14}$. LENS therapy modulates the brain wave frequencies resulting in improvement in socialization and ADL skills.

\section{Fetal Alcohol Spectrum Disorders (FASD)}

Fetal Alcohol Spectrum Disorders (FASD) is a condition that may occur in a new born child whose mother consumed alcohol during pregnancy. The affected child suffers from physical, behavioral and learning problems. D. Corydon Hammond found that LENS therapy shows significant effects behavioral and academic improvements in children with FASD.

\section{Fatigue in Cancer Survivors}

An online survey was conducted by Marian LuctkarFlude and fellows on Neurofeedback providers to determine the protocol used and results. $10 \%$ of the ninety two Neurofeedback providers enrolled in the study used LENS protocol and $37 \%$ of total Neurofeedback providers enrolled reported to apply treatment to cancer survivors. They reported decrease of chronic symptoms in their clients that includes fatigue (16\%), cognitive impairment (23\%/66\%), sleep problems (14\%), Stress (17\%), anxiety (23\%) depression (35\%) and pain (10\%) ${ }^{15}$

\section{Dyslexia}

Dyslexia is also referred as reading disability that occurs when an individual has significant difficulty with speed and accuracy of word decoding. The dyslexic child has difficulty in comprehension of the text and spelling errors. LENS therapy improves academic performance of dyslexic children as it makes easier to process sights and sound, to organize, memorize and retire them.

\section{DISCUSSION}

Although LENS is being exercised around the world, it is still unidentified by many professionals in Pakistan. Prevalence of Mental Health disorders is increasing tremendously in Pakistan. According to WHO more than 14 million people suffer from mild to moderate diagnosed cases of mental illness. The ratio of mental health professional to the population is very low being 203.07 per 100,000 beneficiaries. The expenditure on mental health services in Pakistan is very low. Only $0.4 \%$ of health care budget is expended on mental health care. By the present statistics, the rate of beneficiaries can be estimated to be very high. The other major reason of unpopularity of LENS in Pakistan is lack of technology. The promotion of technology and joint efforts of biomedical engineers and mental health professionals can bring revolution in the mental health care. 


\section{CONCLUSION}

LENS is a type of Neurofeedback that is gentle and noninvasive approach to heal the symptoms of mental health disorders leading to improved quality of life. It transfers very low electromagnetic signals to the brain by its electrodes. Many researchers have supported the benefits of LENS, as of yet, it is unknown to many professionals in Pakistan. The prospect of mental health rehabilitation in Pakistan will increase many folds by introducing and using LENS adjacent to ongoing treatment modalities.

\section{REFERENCES}

[1] Marzbani H, Marateb HR, Mansourian M. Neurofeedback: a comprehensive review on system design, methodology and clinical applications. Basic and clinical neuroscience. 2016;7(2):143.

[2] Liu KK, Bartsch RP, Lin A, Mantegna RN, Ivanov PC. Plasticity of brain wave network interactions and evolution across physiologic states. Frontiers in neural circuits. 2015; 9:62.

[3] Roohi-Azizi M, Azimi L, Heysieattalab S, Aamidfar $M$. Changes of the brain's bioelectrical activity in cognition, consciousness, and some mental disorders. Med. j. Islam. Republran. 2017; 31:53.

[4] Kropotov JD. Quantitative EEG, event-related potentials and neurotherapy. Academic Press; 2010 Jul 28. Harmony T. The functional significance of delta oscillations in cognitive processing. Frontiers in integrative neuroscience. 2013; 7:83.

[5] Harmony T. The functional significance of delta oscillations in cognitive processing. Frontiers in integrative neuroscience. 2013; 7:83.

[6] Jensen MP, Adachi T, Hakimian S. Brain oscillations, hypnosis, and hypnotizability. American
Journal of Clinical Hypnosis. 2015; 57(3):230-53.

[7] Makada T, Ozair D, Mohammed M, Abellanoza $C$. Enhancing memory retention by increasing alpha and decreasing beta brainwaves using music. In Proceedings of the 9th ACM International Conference on Pervasive Technologies Related to Assistive Environments 2016 (pp. 1-4).

[8] Dustman RE, Boswell RS, Porter PB. Beta brain waves as an index of alertness. Science. 1962;137(3529):533-4

[9] Jia X, Kohn A. Gamma rhythms in the brain. PLoS biology. 2011;9(4)

[10] Ochs L, inventor; Ochslabslnc, assignee. Sequential low energy neurofeedback treatment. United States patent US 8,239,014. 2012.

[11] Hammond CD, editor. LENS: The low energy neurofeedback system. Routledge; 2013,11 .

[12] Gillham S, Wild H, Bayer Z, Mitchell M, Sandberg-Lewis K, Colbert A. P02. 89. Low Energy Neurofeedback System (LENS) for stress, anxiety, and cognitive function: an exploratory study. BMC complementary and alternative medicine. 2012; 12(1):P145.

[13] McCarthy-Jones S. Taking back the brain: could neurofeedback training be effective for relieving distressing auditory verbal hallucinations in patients with schizophrenia?. Schizophrenia bulletin. 2012 ;38(4):678-82.

[14] Kennedy-Hendricks A, Epstein AJ, Mandell DS, Candon MK, Marcus SC, Xie M, Barry CL. Effects of state autism mandate age caps on health service use and spending among adolescents. J AmAcad Child Adolesc2018;57(2):125-31.

[15] Luctkar-Flude M, Groll D, Tyerman J. Using neurofeedback to manage long-term symptoms in cancer survivors: Results of a survey of neurofeedback providers. European Journal of Integrative Medicine. 2017; 12:172-6. 